\title{
Modificación de la práctica odontológica, seguimiento a protocolos y percepción de riesgo de los odontólogos durante la pandemia de COVID- 19 en Colombia: estudio de corte transversal
}

\author{
DIANA BARBOSA-LIZI , ANDRÉS A. AGUDELO-SUÁREZ², MARÍA FERNANDA ATUESTA-MONDRAGÓN ${ }^{3}$, JOAQUÍN T. ARIZA-OLAYA4,
} SONIA PATRICIA PLAZA-RUÍZ ${ }^{5}$

\begin{abstract}
${ }^{1}$ Odontóloga. Especialista en Estomatología Pediátrica y en Ortodoncia. Magíster en Educación. Profesora Titular. Posgrado de Ortopedia Maxilar. Posgrado de Ortodoncia. Grupo de Investigación GIONORTO. Facultad de Odontología. Universidad de Antioquia. ORCID: 0000-0003-3679-1142

${ }^{2}$ Odontólogo. Especialista en Administración de Servicios de Salud. PhD en Salud Pública. Profesor Titular. Grupo de Investigación Periodoncia, Educación y Salud. Facultad de Odontología. Universidad de Antioquia. ORCID: 0000-0002-8079-807X

${ }^{3}$ Odontóloga. Especialista en Endodoncia. Presidente Federación Odontológica Colombiana (FOC). Presidente de la Asociación de Profesiones de la salud (ASSOSALUD). Directora Ejecutiva de la Federación Odontológica Latinoamericana (FOLA). Consejera de la Federación Dental Internacional (FDI). ORCID: 0000-0001-6506-672X

${ }^{4}$ Odontólogo. Especialista en Ortodoncia. ORCID: 0000-0003-0561-3423

${ }^{5}$ Odontóloga. Especialista en Ortodoncia. Magíster en Epidemiología. Profesora Asociada, Departamento de Ortodoncia, Fundación Universitaria CIEOUniCIEO. Bogotá, Colombia. ORCID: 0000-0002-4577-3096
\end{abstract}

\section{Resumen}

Introducción: este estudio tuvo como objetivo evaluar la modificación de la práctica, uso de elementos de protección personal, protocolos de bioseguridad, afectación en los planes de carrera, prevalencia de contagio y percepción de riesgo de los odontólogos durante la pandemia de COVID-19 en Colombia. Métodos: tras obtener la aprobación ética, se aplicó una encuesta digital anónima a los odontólogos de Colombia. Las preguntas incluyeron cuatro dominios: 1) sociodemográficos, 2) laborales y afectación en los planes de carrera, 3) Seguimiento a protocolos y bioseguridad y 4) Conocimientos, riesgo y percepción de riesgo de contagio. Se presenta un análisis descriptivo de los datos. Resultados: 5370 odontólogos generales y especialistas participaron del estudio (mujeres: 3878; mediana de edad: 45 años). El 41,94\% fueron odontólogos generales. La adherencia a protocolos de seguridad y el uso de elementos de protección personal (EPP) fue mayor al 99\%. La mayoría de odontólogos $(91,29 \%)$ suspendió sus actividades clínicas durante la cuarentena y ha pensado en disminuir sus horas de trabajo (77,96\%). La percepción de riesgo de contagio de la COVID-19 fue alta (95,91\% refirió que es muy probable/probable el contagio), aunque el auto-reporte de contagio por COVID-19 fue bajo (0,61\%). Conclusión: la pandemia de la COVID-19 ha impactado fuertemente la práctica de la odontología en Colombia, generando cambios en las actividades clínicas y en las perspectivas de carrera. El seguimiento de protocolos de bioseguridad y de uso de EPP fue alto. Aunque la percepción de riesgo de contagio fue alta, el contagio auto-reportado fue muy bajo.

Palabras clave: COVID-19, odontología, consultorios odontológicos, contención de riesgos biológicos

Submitted: September 24/2020 - Accepted: November 03/2020

How to quote this article: Barbosa-Liz D, Agudelo-Suárez AA, Atuesta-Mondragón MF, Ariza-Olaya JT, Plaza-Ruíz SP. Modificación de la práctica odontológica, seguimiento a protocolos y percepción de riesgo de los odontólogos durante la pandemia de COVID-19 en Colombia: estudio de corte transversal. Rev Fac Odontol Univ Antioq. 2021; 33(1): pp-pp. DOI: http://dx.doi.org/10.17533/udea.rfo.v33n1a2 


\section{INTRODUCCIÓN}

Desde finales de 2019 e inicios de 2020 algunos reportes hablaban de la aparición en China de un virus, SARS-CoV-2, que producía un síndrome respiratorio severo agudo denominado la COVID-19 ${ }^{1,2}$. Aunque la COVID-19 ataca fundamentalmente al sistema respiratorio, también se ha visto que presenta manifestaciones en diferentes órganos y sistemas, mostrando alteraciones de la coagulación ${ }^{3}$, del gusto, del olfato, alteraciones dermatológicas e incluso lesiones intraorales ${ }^{4}$. La COVID-19 se fue extendiendo y comenzó a afectar a más países, mostrando altas tasas de contagio, complicaciones y mortalidad. ${ }^{5} \mathrm{~A}$ partir de marzo de 2020, la Organización Mundial de la Salud (OMS), declaró a la infección como una pandemia y los países tuvieron que tomar medidas extremas para tratar de controlar su propagación. ${ }^{5-}$

${ }^{8}$ Esta situación afectó en gran medida a los servicios de salud ${ }^{9-11}$ y la odontología no estuvo exenta de esto. $^{12-14}$

Esta pandemia ha ocasionado hasta la fecha, más de 31 millones de contagiados en el mundo y más de 960 mil muertes, según datos del "CoronaVirus Research Center" de la Universidad John Hopkins para el 21 de septiembre del 2020. ${ }^{15}$ En Colombia, según los datos proporcionados por el Instituto Nacional de Salud, para esta misma fecha, se confirmaban unos 765,076 casos confirmados y 24,208 fallecidos, con una tasa de letalidad de $3.2 \% .{ }^{16}$ De igual forma, hasta el 21 de septiembre del 2020 se han reportado 90 casos de personal odontológico positivos para la COVID-19. ${ }^{16}$ En Colombia, el primer caso de COVID-19 fue reportado el 6 de marzo de 2020. El Ministerio de Salud, expidió diferentes comunicados, reglamentaciones y lineamientos con el fin de controlar la propagación del virus. El 12 de marzo de 2020 se declaró la emergencia sanitaria por el coronavirus COVID-19 y se tomaron gestiones para atender la COVID-19, aún sin restricciones para la prestación de los servicios de odontología. El 25 de marzo se declaró el aislamiento preventivo obligatorio en todo el país y a partir de esta fecha sólo se permitió la atención odontológica de urgencias. ${ }^{17}$ El 14 de mayo el Ministerio de Salud y Protección Social emitió el "lineamiento de bioseguridad para la prestación de servicios relacionados con la atención de la salud bucal durante el periodo de la pandemia por SARS-CoV-2 (COVID-19)" por medio del cual se dictaron las normas y recomendaciones de procesos de bioseguridad y uso de equipos de protección personal (EPP) necesarios durante la atención de urgencias y procedimientos prioritarios en salud oral. ${ }^{18}$ 
Desde la declaración mundial de la pandemia el Ministerio de salud y protección social del país ha emitido diversos lineamientos, protocolos y orientaciones como, los lineamientos de limpieza y desinfección en servicios de salud ante la introducción del nuevo coronavirus (SARS-CoV-2) a Colombia (16 de marzo de 2020) ${ }^{19}$, el plan de acción para la prestación de servicios de salud durante las etapas de contención y mitigación de la pandemia SARS-CoV-2 (COVID-19) (30 de marzo de 2020) y el consenso técnico sobre las condiciones de bioseguridad frente al SARS-CoV-2 y la prevención de la enfermedad COVID-19 en el Sector Salud (Mayo de 2020) ${ }^{20}$, entre otros. A partir del 5 de mayo, el Ministerio de Salud permitió no solo la atención de urgencias sino la atención odontológica prioritaria con protocolos especiales de bioseguridad expedidos en los documentos de: pautas de bioseguridad para la prestación de servicios relacionados con la salud bucal durante el período de la pandemia SARS-COV-2 (covid-19) ${ }^{18}$ y lineamientos para la restauración de los servicios de salud en las fases de mitigación y control de la emergencia sanitaria por Covid-19 en Colombia ${ }^{21}$ (Junio de 2020). Esta situación ha impactado fuertemente a los trabajadores de salud oral (TSO) e incluso ha obligado a muchos de los odontólogos del país a cerrar sus consultas, trayendo como resultado grandes afectaciones en la atención se servicios de salud bucal y en la economía del gremio. ${ }^{14}$

Desde hace muchos años y debido a que, durante los procedimientos odontológicos, existe un contacto cercano con los pacientes que podrían ocasionar el contacto con saliva o sangre contaminada con diversos patógenos, la profesión odontológica ha trabajado continuamente en el mejoramiento de los protocolos de bioseguridad. ${ }^{22}$ El riesgo de infecciones por diversos microrganismos, como el $\mathrm{VIH}$, la hepatitis B, la tuberculosis, entre otros, han sido retos que la odontología ha enfrentado con éxito durante décadas. ${ }^{23,24}$ Sin embargo, con la aparición de la pandemia de COVID-19, los gobiernos, las diferentes entidades de control y los trabajadores de la salud, incluyendo a los odontólogos, han promovido la implementación de mayores medidas preventivas y de protección aplicadas antes, durante y después de la atención odontológica, con el fin de disminuir el riesgo de contagio tanto de los TSO como de sus pacientes. ${ }^{12,25,26}$ Diversos países han reportado, cambios en la atención odontológica, que han sido implementados para garantizar el mínimo riesgo de contagio y una atención de calidad en servicios de salud bucal para los usuarios durante la pandemia del COVID-19. ${ }^{27-29}$ Los nuevos protocolos de atención incluyen el triage telefónico, el aumento en los elementos de protección personal (EPP), tanto para odontólogos como para personal auxiliar, el uso de enjuagues orales para los pacientes, el 
espaciamiento de citas, el control de aerosoles y muchos procedimientos más, con el fin de controlar la propagación del virus. ${ }^{13,30,31}$ El reto al que enfrentan los TSO es alto y como lo menciona Ternera-Pulido ${ }^{14}$ "se requiere garantizar por un lado, la prestación de los servicios en condiciones de bioseguridad, disponibilidad, y suficiencia y por el otro, la garantía de un trabajo en condiciones de dignidad y justicia para todos los profesionales de la salud oral".

Ante el devastador impacto que ha tenido la pandemia de la COVID-19 en todos los sectores, especialmente el de la salud, es muy importante para los TSO, los gremios y los tomadores de decisiones en salud y políticas públicas, conocer estudios con datos confiables y muestras representativas con el fin de tomar decisiones y crear los planes futuros basados en evidencia científica de calidad. Para nuestro conocimiento a la fecha no hay estudios que indaguen sobre el impacto de la COVID-19 en la odontología en Colombia. Por lo tanto, el objetivo de este estudio fue evaluar la modificación de la práctica, afectación en los planes de carrera, uso de elementos de protección personal, protocolos de bioseguridad, prevalencia auto-reportada de contagio y la percepción de riesgo de los odontólogos durante la pandemia de COVID-19 en Colombia.

\section{MÉTODOS}

Estudio transversal mediante una encuesta anónima sobre el impacto de la pandemia de la COVID-19 en la odontología, entre el 19 de junio al 24 de julio de 2020 a los odontólogos que trabajan en Colombia. El cuestionario, que formó parte de una encuesta global ${ }^{32}$ fue validado (validez de apariencia y de contenido y reproducibilidad) en una prueba piloto.

En Colombia no se conoce el número exacto de odontólogos a la fecha, pero una proyección del número de odontólogos para el año 2019, encontró que podrían ser de 55,993 odontólogos. ${ }^{33}$ Sin embargo, el número de odontólogos registrados en las Secretarías de Salud y que laboraron en el territorio nacional ese mismo año fue de $31,872 .{ }^{34}$ El tamaño de muestra fue calculado de acuerdo a la población de odontólogos registrados para el año 2019 mediante el software OpenEpi (Open source epidemiologic statistics for public health version 3.01, AG Dean, KM Sullivan, MM Soe, Atlanta, GA, USA) y la muestra fue recolectada por conveniencia. Con un nivel de confianza del 99,9\% y una prevalencia del 50\% de 
odontólogos que hubieran detenido su actividad clínica por completo, estimada en la prueba piloto y un $3 \%$ de precisión, una muestra mínima de 2,749 participantes era necesaria. Se incluyeron odontólogos que trabajaban tanto en los sistemas nacionales de salud como en clínicas privadas o públicas. El cuestionario incluyó cuatro dominios (D): D1: aspectos sociodemográficos (sexo, edad, región, región geográfica, desempeño laboral, especialización), D2: aspectos laborales y afectación de planes de carrera, D3: aspectos de seguimiento a protocolos y bioseguridad y D4: aspectos de conocimientos, riesgo de contagio y percepción.

La edad fue determinada como una variable continua, sin embargo, para el análisis estadístico ésta fue recategorizada en 4 grupos de edad: adulto joven (22 a 35 años), adulto (36 a 45 años), adulto maduro (46 a 59 años) y adulto mayor ( $\geq 60$ años).

La encuesta fue aprobada por el Comité de Ética de la Fundación Universitaria CIEO-UniCIEO (número 101, acta 62). Este estudio tuvo en cuenta los principios de investigación en humanos de la Declaración de Helsinki de la Asociación Médica Mundial. Todos los encuestados completaron una pregunta de consentimiento informado incluida en la primera página del cuestionario donde el odontólogo podría negar o aprobar su participación en el estudio. La encuesta fue elaborada y distribuida en cuestionarios de Google Forms, con los ajustes necesarios para evitar que fuera llenada dos veces en el mismo dispositivo y fue distribuida por las asociaciones científicas de odontólogos, escuelas de odontología y casas comerciales de productos dentales como COLGATE vía e-mail y redes sociales.

Uno de los investigadores analizó los datos anónimos y verificó su consistencia y calidad. Los análisis estadísticos se realizaron utilizando STATA 16 (StataCorp, College Station, Texas). Se realizó un análisis descriptivo de las variables cualitativas con frecuencias absolutas y relativas y para la variable continua (edad), se tomó la mediana y el rango por tener distribución no normal. 


\section{RESULTADOS}

\section{Aspectos sociodemográficos}

La fiabilidad test-retest fue alta con un coeficiente kappa de 0.91 a 0.96 (IC: 0.77; 0.96). En total se obtuvieron 5,375 encuestas completadas, sin embargo, se descartaron cinco encuestas por errores en el registro de información, quedando 5,370 encuestas viables. Con un total de 31,872 odontólogos registrados para trabajar en el territorio colombiano, la tasa de respuesta correspondiente de la encuesta fue del $16.84 \%$.

El estudio incluyó odontólogos de todo el país con una edad promedio de 45 años (22 a 82 años) y en su mayoría mujeres (72.22\%). La mayoría provenían de las regiones de Bogotá DC (33.87\%) y el $27.41 \%$ provenían de la región central (Antioquia, Caldas, Quindío, Risaralda, Huila, Caquetá y Tolima). Con relación a su formación el 41.94\% fueron odontólogos generales y el desempeño laboral en consultorio privado lo refirieron el $44.71 \%$ de los encuestados. El $15.53 \%$ de los odontólogos reportaron una ciudad de trabajo diferente a su ciudad de residencia (Tabla 1).

Tabla 1. Características sociodemográficas de la población de estudio

\begin{tabular}{|c|c|c|}
\hline Nombre de la variable & Frecuencia absoluta (n) & Frecuencia relativa (\%) \\
\hline \multicolumn{3}{|l|}{ a. Variables Categóricas } \\
\hline \multicolumn{3}{|l|}{ Sexo } \\
\hline Hombre & 1,491 & 27.77 \\
\hline Mujer & 3,878 & 72.22 \\
\hline \multicolumn{3}{|l|}{ Edad categorizada } \\
\hline Joven adulto (22-35 años) & 1,341 & 24.97 \\
\hline Adulto (36-45 años) & 1,488 & 27.71 \\
\hline Adulto maduro (46-59 años) & 2,111 & 39.31 \\
\hline Adulto mayor ( $\geq 60$ años) & 430 & 8.01 \\
\hline \multicolumn{3}{|l|}{ Región Geográfica } \\
\hline Atlántica & 580 & 10.80 \\
\hline Oriental & 878 & 16.35 \\
\hline Bogotá DC & 1,819 & 33.87 \\
\hline Central & 1,472 & 27.41 \\
\hline Pacífica & 563 & 10.48 \\
\hline Amazonas y Orinoquía & 58 & 1.08 \\
\hline \multicolumn{3}{|c|}{ Ciudad de residencia Vs. Ciudad de práctica principal } \\
\hline Diferente & 834 & 15.53 \\
\hline La misma & 4,536 & 84.47 \\
\hline \multicolumn{3}{|l|}{ Especialidad Odontológica } \\
\hline Odontólogo General & 2,252 & 41.94 \\
\hline Administración en salud & 388 & 7.23 \\
\hline Ciencias básicas & 11 & 0.20 \\
\hline
\end{tabular}




\begin{tabular}{|c|c|c|c|c|}
\hline Cirugía oral & \multicolumn{2}{|c|}{58} & \multicolumn{2}{|c|}{1.08} \\
\hline Cirugía Maxilofacial & \multicolumn{2}{|c|}{91} & \multicolumn{2}{|c|}{1.69} \\
\hline Endodoncia & \multicolumn{2}{|c|}{347} & \multicolumn{2}{|c|}{6.46} \\
\hline Implantología & \multicolumn{2}{|c|}{85} & \multicolumn{2}{|c|}{1.58} \\
\hline Medicina oral & \multicolumn{2}{|c|}{22} & \multicolumn{2}{|c|}{0.41} \\
\hline Odontología forense & \multicolumn{2}{|c|}{10} & \multicolumn{2}{|c|}{0.19} \\
\hline Odontopediatría & \multicolumn{2}{|c|}{292} & \multicolumn{2}{|c|}{5.44} \\
\hline Ortodoncia & \multicolumn{2}{|c|}{982} & \multicolumn{2}{|c|}{18.29} \\
\hline Patología oral y maxilofacial & \multicolumn{2}{|c|}{8} & \multicolumn{2}{|c|}{0.15} \\
\hline Periodoncia & \multicolumn{2}{|c|}{261} & \multicolumn{2}{|c|}{4.86} \\
\hline Prostodoncia/Rehabilitación oral & \multicolumn{2}{|c|}{473} & \multicolumn{2}{|c|}{8.81} \\
\hline Radiología dentomaxilofacial & \multicolumn{2}{|c|}{8} & \multicolumn{2}{|c|}{0.15} \\
\hline Salud pública & \multicolumn{2}{|c|}{82} & \multicolumn{2}{|c|}{1.53} \\
\hline Desempeño laboral & Sí & No & Sí & No \\
\hline Odontólogo privado (propietario) & 2,401 & 2,969 & 44.71 & 55.29 \\
\hline Odontólogo privado (porcentaje y/o arrienda) & 1,926 & 3,444 & 35.87 & 64.13 \\
\hline Odontólogo clínico empleado (en entidad pública o privada) & 1,637 & 3,733 & 30.48 & 69.52 \\
\hline Odontólogo en docencia en una o más universidades & 723 & 4,647 & 13.46 & 86.54 \\
\hline \multicolumn{4}{|l|}{ b. Variables continuas } & 93.07 \\
\hline Nombre de la variable & \multicolumn{2}{|c|}{ Mediana } & \multicolumn{2}{|c|}{ Min-Max } \\
\hline Edad & \multicolumn{2}{|c|}{45} & \multicolumn{2}{|c|}{$22-82$} \\
\hline
\end{tabular}

Fuente: los autores

\section{Aspectos laborales y afectación en planes de carrera}

Al inicio de la declaración del aislamiento social obligatorio solo el $2.33 \%$ siguió trabajando con normalidad. Con relación al reinicio de atención de urgencias presenciales, desde el 14 de mayo, el 63.49\% de los encuestados ya atendían urgencias presenciales y el $36.51 \%$ después de esta fecha. La principal causa para limitar o detener la actividad clínica presencial fue para cumplir los lineamientos del gobierno nacional (65.94\%). Respecto al impacto que ha tenido la COVID-19 en los planes de carrera, el $21.75 \%$ de los encuestados ha pensado en el retiro temprano, mientras que el $77.96 \%$ en reducir las horas de trabajo (Tabla 2).

Tabla 2. Aspectos laborales y modificación de los planes de carrera en la población de estudio

\begin{tabular}{lcc}
\hline \multicolumn{1}{c}{ Nombre de la variable } & Frecuencia absoluta (n) & Frecuencia relativa (\%) \\
\hline $\begin{array}{l}\text { Al inicio de la declaración de aislamiento } \\
\text { social obligatorio en todo el territorio }\end{array}$ & & \\
$\begin{array}{l}\text { nacional colombiano (25 de marzo de } \\
\text { 2020) usted:) }\end{array}$ & & \\
$\begin{array}{l}\text { Continuó trabajando con normalidad } \\
\text { Limitó su actividad clínica a urgencias }\end{array}$ & 125 & 2.33 \\
dentales (presenciales) & 1,002 & 18.66
\end{tabular}


Detuvo totalmente su actividad clínica y sólo atendió urgencias de forma remota (teléfono/video/WhatsApp/Redes

Antes de la declaración oficial de aislamiento social obligatorio (25 de marzo de 2020)

Desde la declaración oficial de aislamiento social obligatorio (25 de marzo de 2020) Desde que el Ministerio de Salud y Protección Social de Colombia, expidió los lineamientos para la atención de urgencias por alteraciones de salud bucal en el marco de la pandemia por el SARS-Cov2 (4de mayo de 2020)

Desde que el Ministerio de Salud y Protección Social, expidió los lineamientos de bioseguridad, para la prestación de servicios relacionados con la atención en salud bucal, en el marco de la pandemia por SARS-Cov2 (14 de mayo de 2020) Posterior a estas fechas

Si en algún momento detuvo su actividad clínica presencial por completo, ¿Desde cuándo lo hizo?

A partir del primer caso de COVID-19 en Colombia (6 de marzo)

401

Días antes de decretarse la cuarentena (25 de marzo)

2,348

50.92

Cuando se inició la cuarentena ( 25 de marzo)

Posterior a estas fechas

170

No aplica $\mathrm{n}=759$

Si en algún momento detuvo o limitó su actividad clínica presencial, ¿Por qué razón lo hizo?

Cerró la clínica o institución en la que trabajo

Sentí inseguridad o miedo ante un posible contagio

No disponía de suficientes elementos de protección personal (EPP)

Pensé que disminuiría la demanda de pacientes debido al confinamiento Pensé que era obligatorio cerrar

Para cumplir con los lineamientos del gobierno nacional al respecto 
No detuvo la actividad clínica

No aplica (no realizo actividad clínica) $n=303$

¿Cómo ha influido el impacto de la

pandemia por COVID-19 en sus planes de

carrera como odontólogo?

Considerar el retiro temprano

Reducir las horas de trabajo

Cambiar la ubicación actual de la práctica

Cambiar a otra carrera diferente a

odontología

Cambiarse a una posición dental no clínica

Sin impacto en los planes de carrera

$\begin{array}{cccc}230 & 4,878 & 4.50 & 95.50 \\ & & & \\ \text { Si } & \text { No } & \text { Si } & \text { No } \\ & & & \\ 1,168 & 3,233 & 21.75 & 78.25 \\ 3,431 & 970 & 77.96 & 22.04 \\ 408 & 3,993 & 9.27 & 90.73 \\ 799 & 3,602 & 18.15 & 81.85 \\ 705 & 3,696 & 16.02 & 83.98 \\ 1,108 & 4,262 & 20.63 & 79.37\end{array}$

Fuente: los autores

\section{Aspectos de seguimiento a protocolos y bioseguridad}

El 100\% de los encuestados sigue una o más medidas de precaución como enjuague bucal preoperatorio con peróxido de hidrógeno al 1\% (99.86\%), desinfección de superficies (93.50\%), lavado de manos (91.67\%), verificación del estado de salud de los pacientes (90.16\%) entre otros. En cuanto al uso de EPP, El del 89.65\% utiliza tapabocas N95 o similares y mascarilla quirúrgica el $43.97 \%$. Bata lavable antifluido el $72.19 \%$ y bata desechable antifluido estéril o no el 100\%, así como guantes desechables estériles (22.89\%) o no (91.45\%) (Tabla 3).

Tabla 3. Medidas de bioseguridad tomadas en la práctica odontológica y uso de elementos de protección personal (EPP)

\section{durante la pandemia}

\begin{tabular}{|c|c|c|c|c|}
\hline$\frac{\text { Nombre de la variable }}{\text { En caso de que usted durante la pandemia }}$ & \multicolumn{2}{|c|}{ Frecuencia absoluta (n) } & \multicolumn{2}{|c|}{ Frecuencia relativa $(\%$} \\
\hline $\begin{array}{l}\text { esté trabajado, ¿Cuáles de las siguientes } \\
\text { medidas de precaución ha tomado? }\end{array}$ & Sí & No & Sí & No \\
\hline Triage telefónico & 3,348 & 820 & 88.33 & 19.67 \\
\hline Espaciamiento de las citas & 3,704 & 464 & 88.87 & 11.13 \\
\hline $\begin{array}{l}\text { Verificación del estado de salud del } \\
\text { paciente antes de citarlo }\end{array}$ & 3,758 & 410 & 90.16 & 9.84 \\
\hline $\begin{array}{l}\text { Posponer las citas en personas mayores o } \\
\text { con patologías sistémicas }\end{array}$ & 2,648 & 1,520 & 36.47 & 63.53 \\
\hline $\begin{array}{l}\text { Toma de temperatura del paciente al } \\
\text { Ilegar al consultorio }\end{array}$ & 3,798 & 370 & 91.12 & 8.88 \\
\hline $\begin{array}{l}\text { Desinfección de superficies varias veces al } \\
\text { día: manijas, interruptores, sala de espera } \\
\text { y recepción, etc. }\end{array}$ & 3,854 & 314 & 92.47 & 7.53 \\
\hline $\begin{array}{l}\text { Desinfección varias veces al día de todos } \\
\text { los elementos y superficies del área clínica }\end{array}$ & 3,897 & 271 & 93.50 & 6.50 \\
\hline $\begin{array}{l}\text { Toma de temperatura a todos los } \\
\text { trabajadores y excluir a aquellos con } \\
\text { temperatura superior a } 37.5^{\circ} \mathrm{C}\end{array}$ & 3,243 & 925 & 77.81 & 22.19 \\
\hline
\end{tabular}


Lavado de manos de los pacientes antes

de entrar al consultorio

\begin{tabular}{|c|c|c|c|}
\hline 3,821 & 347 & 91.67 & 8.33 \\
\hline 3,341 & 827 & 80.16 & 19.84 \\
\hline 3,768 & 400 & 90.40 & 9.60 \\
\hline 1,714 & 2,454 & 41.12 & 58.88 \\
\hline 1,247 & 2,921 & 70.08 & 29.92 \\
\hline 2,072 & 2,096 & 49,71 & 50,29 \\
\hline 3,477 & 691 & 83.42 & 16.58 \\
\hline 2,905 & 1,263 & 69.70 & 30.30 \\
\hline 2,176 & 3 & 99.86 & 0.14 \\
\hline 1,464 & 2,704 & 35.12 & 64.88 \\
\hline 380 & 3,788 & 9.12 & 90.88 \\
\hline 279 & 3,889 & 6.69 & 93.31 \\
\hline 356 & 3,812 & 8.54 & 91.46 \\
\hline 2,659 & 1,509 & 63.80 & 36.20 \\
\hline 2,928 & 1,24 & 70.25 & 29.75 \\
\hline 2,328 & 1,84 & 55.85 & 44.15 \\
\hline 2,459 & 1,709 & 59.00 & 41.00 \\
\hline 3,965 & 655 & 85.82 & 14.18 \\
\hline 3,574 & 594 & 85.75 & 14.25 \\
\hline
\end{tabular}

Mantener al menos 1 metro de distancia

entre los pacientes

Ofrecer o solicitar el uso de tapabocas al

paciente mientras espera

Polainas para cubrir los zapatos de los

pacientes

Colocar divisiones entre unidades

odontológicas

Ventilación frecuente de los espacios o

salas de espera

Eliminación de revistas y/o folletos de las

salas de espera

Dejar abrigos, bolsos u otros objetos

personales de los pacientes fuera del área

clínica

Enjuague bucal preoperatorio con

peróxido de hidrógeno al $1 \%$

Enjuague bucal preoperatorio con

clorhexidina al 0.12-0.2\%

Enjuague bucal preoperatorio con

povidona yodada al $0.2-1 \%$

Enjuague bucal preoperatorio con cloruro

de cetilpiridinio al $0.05-0.10 \%$

Enjuague con enjuague bucal diluido

Ventilación del área clínica al menos 10

minutos entre pacientes

Desinfección de superficies con alcohol al

$70 \%$

Desinfección de superficies con hipoclorito

de sodio al 0,5\%

Otros desinfectantes comunes de clínica

Lavado de manos del operador antes y

después de cada procedimiento

Retirada de todos los dispositivos de

protección desechables y desinfección de

los no desechables

durante la pandemia) $n=1,241$

No aplica (no trabajo en clínica o no he trabajado durante la pandemia) n=1,241

¿Cuáles de los siguientes elementos de

protección (EEP) ha utilizado usted durante

Sí

la atención clínica?

Tapabocas quirúrgico

Tapabocas N95, FFP2 o FFP3

Respiradores de Silicona (media cara o

cara completa)

Gorros desechables

Bata estéril antifluido desechable

Bata lavable antifluido

Bata desechable antifluido

Overol de protección

Gafas o Monogafas

Careta facial (Visor)

$\begin{array}{cccc}\text { Sí } & \text { No } & \text { Sí } & \text { No } \\ & & & \\ 2,399 & 1,883 & 56.03 & 43.97 \\ 3,839 & 443 & 89.65 & 10.35 \\ 558 & 3,724 & 13.03 & 86.97 \\ 3,764 & 518 & 87.90 & 12.10 \\ 1,381 & 2.901 & 67.75 & 32.25 \\ 3,091 & 1,191 & 72.19 & 27.81 \\ 1,535 & 2,747 & 35.85 & 64.15 \\ 2,309 & 1,973 & 53.92 & 46.08 \\ 3,391 & 891 & 79.19 & 20.81 \\ 4,122 & 160 & 96.26 & 3.74\end{array}$




$\begin{array}{lcccc}\text { Guantes estériles desechables } & 980 & 3,302 & 22.89 & 77.11 \\ \text { Guantes desechables } & 3,916 & 366 & 91.45 & 8.55 \\ \text { Instrumentos rotatorios con válvula } & 973 & 3,309 & 22.72 & 77.28 \\ \text { antiretorno } & 972 & 3,310 & 22.70 & 77.30 \\ \text { Aislamiento con tela de caucho } & 2,540 & 1,742 & 59.32 & 40.68 \\ \text { Polainas } & \end{array}$

No aplica (no trabajo en clínica o no he trabajado durante la pandemia) $n=1,122$

Source: by authors

\section{Aspectos de conocimientos, riesgo de contagio y percepción}

El 80\% reportaron no presentar ningún síntoma asociado con la COVID-19. Un total de 458 odontólogos encuestados (8.5\%) refirió haberse practicado la prueba de la COVID-19, de las cuales solo 33 resultaron positivas $(0.61 \%)$. En cuanto a la percepción de contagio el $69.26 \%$ piensa que es muy probable el contagio en sus labores clínicas (Tabla 4).

Tabla 4. Pruebas de COVID-19 y percepción de riesgo de contagio en la población de estudio

\begin{tabular}{|c|c|c|c|c|}
\hline & \multicolumn{2}{|c|}{ Frecuencia absoluta (n) } & \multicolumn{2}{|c|}{ Frecuencia Relativa (\%) } \\
\hline $\begin{array}{l}\text { ¿Desde la aparición del COVID-19, ha } \\
\text { experimentado algunos de los siguientes } \\
\text { sintomas? }\end{array}$ & Sí & No & Sí & No \\
\hline Dolor de Cabeza & 663 & 4,707 & 12.35 & 87.65 \\
\hline Congestión nasal & 284 & 5,086 & 5.29 & 94.71 \\
\hline Tos & 236 & 5,134 & 4.39 & 95.61 \\
\hline Dolor generalizado & 103 & 5,217 & 1.92 & 98.08 \\
\hline Dolor de garganta & 375 & 4,995 & 6.98 & 93.02 \\
\hline Fatiga & 188 & 5,182 & 3.50 & 96.50 \\
\hline Diarrea & 139 & 5,231 & 2.59 & 97.41 \\
\hline Conjuntivitis & 57 & 5,313 & 1.06 & 98.94 \\
\hline Dificultad respiratoria & 51 & 5,319 & 0.95 & 99.05 \\
\hline Pérdida del olfato & 50 & 5,32 & 0.93 & 99.07 \\
\hline Pérdida del gusto & 46 & 5,324 & 0.86 & 99.14 \\
\hline Fiebre & 85 & 5,285 & 1.58 & 98.42 \\
\hline Sangrado nasal & 16 & 5,354 & 0.30 & 99.70 \\
\hline Sin síntomas & 4,302 & 1,068 & 80.11 & 19.89 \\
\hline \multicolumn{5}{|c|}{ ¿Le han hecho a usted la prueba para COVID-19? } \\
\hline 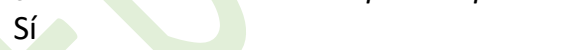 & \multicolumn{2}{|c|}{458} & \multicolumn{2}{|c|}{8.53} \\
\hline No & \multicolumn{2}{|c|}{4,912} & \multicolumn{2}{|c|}{91.47} \\
\hline \multicolumn{5}{|c|}{ ¿Cuál fue el resultado de su prueba de COVID -19?) } \\
\hline COVID-19 Positivo & \multicolumn{2}{|c|}{33} & \multicolumn{2}{|c|}{0.61} \\
\hline COVID-19 Negativo & \multicolumn{2}{|c|}{422} & \multicolumn{2}{|c|}{7.86} \\
\hline No aplica (no me han hecho la prueba) & \multicolumn{2}{|c|}{4,915} & \multicolumn{2}{|c|}{91.53} \\
\hline \multicolumn{5}{|c|}{ ¿Ha realizado algún curso (con o sin certificado) sobre COVID-19? } \\
\hline Sí & \multicolumn{2}{|c|}{3,498} & \multicolumn{2}{|c|}{65.14} \\
\hline No & \multicolumn{2}{|c|}{1,812} & \multicolumn{2}{|c|}{34.86} \\
\hline \multicolumn{5}{|c|}{ ¿Cree que posee conocimientos suficientes sobre COVID-19? } \\
\hline $\mathrm{Si}$ & \multicolumn{2}{|c|}{3,688} & \multicolumn{2}{|c|}{68.88} \\
\hline
\end{tabular}




\begin{tabular}{|c|c|c|}
\hline No & 1,671 & 31.12 \\
\hline \multicolumn{3}{|c|}{ ¿Cree usted que la infección COVID-19 es un riesgo para los odontólogos? } \\
\hline Muy Probable & 3,719 & 69.26 \\
\hline Probable & 1,431 & 26.65 \\
\hline Poco probable & 207 & 3.85 \\
\hline Improbable & 13 & 0.24 \\
\hline \multicolumn{3}{|c|}{ Cree usted que el riesgo de transmisión de COVID-19 durante la práctica odontológica es: } \\
\hline Mayor que ir al supermercado & 3,167 & 58.98 \\
\hline Comparable con ir al supermercado & 732 & 13.63 \\
\hline Menor que ir al supermercado & 1,471 & 27.39 \\
\hline \multicolumn{3}{|c|}{ ¿Cuánta confianza tiene usted de que no se contagiará por COVID-19 durante su actividad clínica? } \\
\hline Plena confianza & 493 & 9.18 \\
\hline Suficiente confianza & 2,961 & 55.14 \\
\hline Poca confianza & 1,603 & 29.85 \\
\hline Ninguna confianza & 313 & 5.83 \\
\hline
\end{tabular}

Source: by authors

\section{DISCUSIÓN}

\section{Aspectos Sociodemográficos}

La presente investigación describe las modificaciones en la práctica, en los planes de carrera, el desempeño laboral, riesgo de contagio, uso de EPP y procedimientos de bioseguridad que han realizado los odontólogos en Colombia durante el brote de la COVID-19. Nuestra muestra estuvo compuesta en un porcentaje muy alto de mujeres (72.22\%), lo cual puede estar relacionado con la mayor proporción de mujeres odontólogas en Colombia. Berdejo-Casas J y Ternera-Pulido J. ${ }^{34}$ reportaron en su informe para la Alcaldía Mayor de Bogotá, que de los odontólogos registrados en el país para marzo del 2019, el 75.46\% fueron mujeres. En cuanto a la edad se observó una representación en todos los grupos etarios en la muestra, con mayor porcentaje del grupo entre los 46 a 59 años (39.31\%) y un menor porcentaje de adultos mayores ( $\geq 60$ años; 8.01\%). Según el registro especial de prestadores en salud (REPS) en un informe del 2015, los valores observados en este estudio fueron similares a los grupos de edad de los odontólogos registrados en esa fecha. ${ }^{34}$ La mayor representación de las regiones fue en Bogotá (33.87\%) y la región central de Colombia (27.41\%); esto podría deberse a la tendencia nacional de concentración de odontólogos en zonas urbanas con mayor acceso a internet y redes sociales o podría deberse a un mayor número de odontólogos en estas zonas urbanas que tienen buen acceso a las redes sociales, medio por el cual la encuesta fue distribuida. Según un informe del Ministerio de Salud para la 
Federación Odontológica Colombiana (FOC) de septiembre del 2020,35 en Colombia la proyección de odontólogos por municipios muestra porcentajes similares de concentración de odontólogos en las ciudades y municipios a los que encontramos en nuestra encuesta. Por lo anterior podría considerarse que la muestra del presente estudio fue representativa de los odontólogos que trabajan en Colombia.

De las especialidades de los odontólogos que respondieron la encuesta se encontró una mayor proporción de odontólogos generales (41.94\%) seguidos de ortodoncistas (18,29\%), rehabilitadores orales (8,81\%), administración en salud (7.23\%), endodoncia (6.46\%), odontopediatría (5.44\%) y periodoncia (4.86\%). Según el REPS en Colombia a 31 de marzo del 2020, el mayor número de registros correspondió a odontólogos generales $(20,651)$, seguidos por ortodoncistas $(5,928)$, endodoncistas $(2,744)$, periodoncistas $(2,430)$, rehabilitación oral $(2,423)$ y odontopediatría $(1,546) .{ }^{14}$ Aunque es importante anotar de que hay un subregistro de los profesionales de la salud oral ante el Ministerio de Salud, las proporciones podrían mantenerse. El alto número de respuestas de ortodoncistas también puede deberse a que dos de las investigadoras son ortodoncistas y podrían haber tenido mayor influencia entre sus colegas para responder la encuesta, así mismo la Sociedad Colombiana de Ortodoncia realizó gran acompañamiento en la divulgación de la encuesta entre sus miembros.

En cuanto al desempeño laboral solo el $44.4 \%$ de los encuestados son odontólogos privados propietarios de sus consultorios o clínicas y el $35.87 \%$ trabajan por porcentaje o arriendan. Un $30.48 \%$ son empleados, un $13 \%$ académicos y $6.93 \%$ trabajan en áreas administrativas. Ternera-Pulido J. ${ }^{33}$ encontró que entre los que ofertan servicios de salud oral en Colombia, el 57\% de los registrados en el Ministerio de Salud para el año 2018 eran profesionales independientes y el 43\% eran instituciones prestadoras de salud (IPS) públicas o privadas. Adicionalmente, en nuestro estudio el $15.53 \%$ de los encuestados refirió trabajar en una ciudad o municipio diferente al lugar de residencia. Este último resultado, haría pensar que este porcentaje de odontólogos han estado altamente afectados por la actual pandemia, en la prestación de los servicios de salud oral, ya que en el país los viajes aéreos nacionales solo fueron reactivados en septiembre y por carretera los viajes interdepartamentales también estuvieron restringidos un periodo de tiempo similar. Así mismo las comunidades que en su mayoría habitan en áreas rurales o remotas donde estos odontólogos prestaban sus servicios antes de la pandemia del 
COVID-19, pudieron haber quedado desprotegidas en la atención en salud oral, lo cual puede causar un impacto actual y futuro en la profundización de las inequidades en salud oral del país. ${ }^{14}$

\section{Aspectos laborales y afectación en planes de carrera}

A partir de la declaración de cuarentena nacional en Colombia el 25 de marzo del 2020 a causa del brote de COVID-19 en el país, los servicios de atención en salud oral fueron suspendidos en todo el territorio nacional y solo se permitió la atención de urgencias dentales. Durante ese tiempo, solo el 18,66\% de los encuestados atendió urgencias mientras que el 79.01\% detuvo completamente su actividad clínica presencial. Solamente el $35,08 \%$ atendió urgencias antes del 4 de mayo y el $36.51 \%$ de los encuestados inicio atención clínica de urgencias después del 14 de mayo del 2020. Casi el 60\% de los encuestados detuvo su consulta clínica presencial antes de decretarse la cuarentena y el 95.5\% detuvieron o limitaron su actividad clínica desde marzo hasta mayo del 2020 o incluso más tiempo. Este porcentaje reportado es particularmente alto si lo comparamos con los porcentajes encontrados por Tysiąc-Miśta M y Dziedzic A. ${ }^{13}$ en su estudio en Polonia (71.2\%), Ahmed and Jouhar et al. ${ }^{36}$ en un estudio de 650 odontólogos de diversos países (66\%) y Duruk G et al. ${ }^{37}$ (12.30\%) en Turquía. El alto porcentaje de cese de las actividades laborales de los odontólogos encontrado en nuestro estudio puede deberse a que las regulaciones gubernamentales en Colombia fueron especialmente restrictivas en la atención en salud oral, a la tardía emisión de los lineamientos de bioseguridad para la atención de urgencias en salud oral y/o al miedo de los profesionales de contagiarse o contagiar a sus familias. ${ }^{36}$

En cuanto a las causas de la detención de la actividad clínica presencial el 65\% lo hizo para cumplir con los lineamientos del gobierno nacional al respecto, el 36\% porque sintió inseguridad, un 17\% porque no disponía de suficientes elementos de EPP y el 27\% porque cerro la clínica o institución donde trabajaban y solamente un $4.5 \%$ no detuvo o limitó su actividad clínica. En otros países los hallazgos a este respecto son similares, Tysiąc-Miśta $\mathrm{M}$ y Dziedzic $A{ }^{13}$ encontraron que la principal causa para descontinuar la atención clínica fue el miedo por su propio bienestar y el de sus familias ( 51.2 y $57.6 \%$ respectivamente), una segunda razón fue la falta de EPP (63.4\%) y otros por regulaciones del gobierno (53.5\%). En su investigación, Duruk et al. ${ }^{37}$ y Ahmed MA et al. ${ }^{36}$ reportaron que más del $90 \%$ de los odontólogos encuestados estaban preocupados por su seguridad y la de sus familias. 
La situación laboral en odontología en Colombia ya era muy compleja antes de la pandemia de la COVID19 debido a la flexibilización laboral, competencia desleal en precios de clínicas de cadena, altos costos y presión psicológica que acarrea la habilitación de los consultorios y que desmotiva la consulta privada por lo que aproximadamente el $60 \%$ de los odontólogos trabajaban para terceros, siendo sometidos a malas condiciones laborales lo que disminuye su bienestar material y seguridad económica. ${ }^{14} \mathrm{Sin}$ embargo, ante el cese laboral y la limitación del ejercicio por las regulaciones gubernamentales, la inseguridad y miedo al contagio, el cierre de sus lugares de trabajo y la falta de disponibilidad y los altos costos de elementos de protección personal (EPP) por el brote de la COVID-19 en el país, la situación laboral actual de los odontólogos privados que solo devengan cuando atienden pacientes sin un sueldo fijo es devastadora y seguramente tendrá fuertes repercusiones en la comunidad odontológica, si los entes gubernamentales y gremios no toman acciones inmediatas. La estimación de desempleo entre los odontólogos en Colombia para el 2020 era de un 30\% sin tener en cuenta la pandemia de la COVID-19. ${ }^{33}$ Según el Departamento Administrativo Nacional de Estadística (DANE) en julio del 2020 en Colombia el desempleo se posicionó en el 20.2\% lo que significó un aumento de 9.5 puntos porcentuales frente al mismo mes del año anterior (10.7\%). Este incremento del desempleo se dio por las medidas de aislamiento tomadas para contener la pandemia de la COVID-19 que golpearon fuertemente la economía y así mismo al mercado laboral en el sector de la odontología. ${ }^{38}$ La economía en el sector de la odontología no solo afecta a los odontólogos sino también a los recursos humanos, industria y comercio que gira alrededor de ellos, como auxiliares, laboratoristas dentales, casas comerciales y fábricas de unidades y productos odontológicos. Ternera-Pulido J. ${ }^{14}$ determinó que para el año 2014, en Colombia el sector de la odontología, movía anualmente un total de \$2,138,220,000,000 (dos billones, ciento treinta y ocho mil doscientos veinte millones de pesos). Basado en estos datos, el administrador de empresas Mauricio Martínez Pinto realizó una proyección económica para la FOC basado en el índice de precios al consumidor (IPC) del 30.48\%, conseguido con la proyección de los valores del 2014 a cinco años (2014-2019). El encontró que el valor indexado para el gasto mensual que generaba el sector de la odontología a finales del 2019 estaba alrededor de $\$ 232,495,124,621$ (doscientos treinta y dos mil cuatrocientos noventa y cinco millones ciento veinticuatro mil seiscientos veintiún mil de pesos), lo que anualmente correspondería a $\$ 2,789,941,495,450$ (dos billones setecientos ochenta y nueve mil novecientos cuarenta y un mil millones cuatrocientos noventa y cinco mil cuatrocientos cincuenta pesos. 
Si tomamos lo anterior como referencia podríamos estar hablando de que las pérdidas en casi tres meses de detener la actividad clínica en el sector odontológico podría ser una cifra superior a los $\$ 700,000,000,000$ (setecientos mil millones de pesos) y teniendo en cuenta que, algunas de las actividades a la fecha de la escritura de este articulo (septiembre 2020) siguen restringidas, las pérdidas económicas en el sector serán mucho mayores.

En cuanto al impacto de la pandemia de COVID-19 en los planes de carrera como odontólogo, 79.37\% de los encuestados respondieron que ésta tuvo algún impacto como el reducir sus horas de trabajo (77, $+.96 \%)$, considerar retirarse temprano (21.75\%), cambiarse a una carrera diferente a la odontología (18.15\%), moverse a una posición no clínica (16.02\%) y cambiar la ubicación de su práctica (9.27\%). Ternera-Pulido J. ${ }^{14}$ encontró que según el REPS, hubo una disminución de los prestadores de salud oral habilitados de marzo del 2020 a Julio del 2020 que pasó de 38,216 prestadores registrados a 37,450, aunque esta reducción corresponde solo al $2 \%$ de los prestadores habilitados, en algunas especialidades como la cirugía oral se pasó de 1,265 prestadores registrados a 201, lo que significa una reducción cercana al $84 \%$. Los resultados de nuestro estudio muestran el gran impacto de la actual pandemia, no solo en los aspectos laborales actuales sino también en sus planes futuros, por lo que si no hay políticas públicas contundentes, planes de préstamos de fácil acceso y bajos intereses, baja en la carga y costos de los procesos de habilitación para motivar a los odontólogos a mantener sus consultas, es probable que en muy poco tiempo la crisis en el sector odontológico será muy grande y por ende esto tendrá repercusión en la salud oral de los colombianos. La Federación Dental Internacional (FDI) tenía dentro de la visión de la odontología para el 2020 "reducir sustancialmente las desigualdades en el acceso a la atención odontológica y a cubrir más ampliamente las necesidades y la demanda mundial de atención bucodental". ${ }^{39}$ Sin embargo, ante la situación actual de la odontología en el mundo a causa de la pandemia de la COVID-19 estos planes serán muy difíciles de cumplir sobre todo en países en vía de desarrollo y sin planes particulares de ayuda a los TSO, como Colombia.

\section{Aspectos de seguimiento a protocolos y bioseguridad}

Ante la contingencia de la pandemia por la COVID-19, diversos protocolos y manuales de bioseguridad han sido realizados tanto por las entidades gubernamentales de Colombia como el Ministerio de Salud y también por la comunidad científica. ${ }^{18-20,40,41}$ En todos ellos hay un consenso de las medidas de 
precaución que el personal de salud debe tomar para disminuir el riesgo de contagio del virus del SARSCov-2. De acuerdo al documento expedido por el Ministerio de Salud y Protección Social de agosto del 2020 donde reúne todos los lineamientos orientaciones y protocolos para enfrentar la COVID-19 en Colombia, la recomendación para el personal de salud de los EPP básicos que debe usar el talento humano en salud dependiendo de la generación de aerosoles son gorro, careta facial, respirador N95, guantes desechables, uniforme antifluido, bata antifluido, polainas o calzado especial si realiza procedimientos que generan aerosoles. Si se realizan procedimientos no generadores de aerosoles la recomendación es la misma pero se cambian dos elementos, monogafas (en lugar de careta facial) y mascarilla quirúrgica (en lugar del respirador N95). ${ }^{20,42}$ En nuestra investigación el $90 \%$ de los odontólogos refirieron estar usando respiradores N95, FFP2 o FFP3, un 56\% mascarillas quirúrgicas y un $13 \%$ respiradores de silicona. Cagetti et al. ${ }^{27}$ reportaron que 55\% de los encuestados usaba respiradores FPP2 o FPP3. Otros estudios ${ }^{36,37}$ han reportado que solo cerca del $10 \%$ de los odontólogos encuestados usaban respirador N95 en la atención clínica, lo que contrasta con nuestros resultados en el que el $90 \%$ de los odontólogos refieren utilizarlo.

En cuanto a protección ocular en nuestra investigación, un 96\% respondió que usan careta facial, 79\% gafas o monogafas. Respecto a la indumentaria utilizada, fue alto el número de odontólogos que refirieron el uso de bata antifluido tanto desechable (no estéril $35.85 \%$ y estéril $67.75 \%$ ) como no desechable (72.19\%) y gorro desechable (87.90\%). Igualmente, el uso de guantes desechables no estériles $(91.45 \%)$ y estériles $(22.89 \%$ ) y polainas $(59.32 \%)$. Estos resultados sugieren una alta adherencia de los odontólogos en Colombia en el uso de los EPP y que incluso en algunos casos utilizan dos elementos para la misma protección por ejemplo gafas o monogafas y careta facial, como pudo observarse en los altos porcentajes de respuesta de uso de estos elementos, debido a que esta pregunta el encuestado podía escoger uno o varios ítems a la vez.

Por otra parte, el uso de tela de caucho y de instrumentos rotatorios con válvula anti-retorno fue baja (22\%), que podría deberse a que la tela de caucho limita las áreas a tratar en procedimientos que se tiene que tener visibilidad de todos los dientes como en los tratamientos de ortodoncia y en cuanto a los instrumentos rotarios con válvula anti-retorno su disponibilidad y costos pueden limitar su uso. 
En nuestro estudio los encuestados refirieron una alta adherencia a los protocolos de bioseguridad con un porcentaje de respuesta mayor del $85 \%$, con el uso de desinfección de superficies, lavado de manos, toma de temperatura corporal, verificación del estado de salud del paciente, modificaciones en los consultorios y enjuague bucal preoperatorio entre otros. Estos resultados pudieron estar relacionados con el amplio conocimiento y adherencia previa a la mayoría de estos procedimientos de bioseguridad que ya eran parte de los requisitos mínimos de habilitación de las Secretarias de Salud nacionales que son de obligatorio cumplimiento para la habilitación de los consultorios odontológicos. Contrario a lo reportado por Duruk et al. ${ }^{37}$ en su estudio entre odontólogos turcos, que aunque toman ciertas medidas de precaución para la COVID-19, estas no son suficientes para su protección, del personal auxiliar y los pacientes. Por otra parte, De Stefani et al. ${ }^{28}$ encontraron que la falta de protocolos específicos crean inseguridad sobre el control de infecciones y el uso de EPP durante la práctica odontológica en la actual pandemia. Adicionalmente, Singh Gambhir R. et al. ${ }^{29}$ encontraron que entre un $86.2 \%$ y $94.2 \%$ de los odontólogos encuestados seguían prácticas de desinfección de superficies y lavado de manos en sus actividades clínicas.

También es importante considerar aquí, el aumento de los costos de consulta para garantizar el uso de EPP y medidas de precaución para la atención clínica en salud oral. Un estudio de costos realizado por la cooperativa de la SCO (SCO life ${ }^{43}$ proyectó que los gastos por insumos básicos de bioseguridad por cita de cada paciente era de $\$ 12,277$. A lo anterior hay que sumarle los sobrecostos actuales de los EPP, de hasta 10 veces más de lo que costaban antes de la pandemia del COVID-19, debido a la escasez de estos elementos en el país. Sería importante que el Gobierno Nacional reconociera los grandes esfuerzos de los TSO en el cumplimiento de los lineamientos de bioseguridad y uso de EPP y creara planes especiales de control de precios de los EPP y elementos de bioseguridad para los odontólogos del país.

\section{Aspectos de conocimientos, riesgo de contagio y percepción de riesgo de la COVID-19}

En el presente estudio el $68.88 \%$ dijo creer que tienen conocimientos suficientes sobre COVID-19 y el $65.14 \%$ refirió haber realizado un curso al respecto. Resultados similares se han encontrado en otros estudios. En Italia los encuestados refirieron en un $84.4 \%$ que tenían adecuado conocimiento sobre la COVID-19, ${ }^{30}$ mientras que en Turquía 69.8\% de los encuestados reportó tener suficiente conocimiento acerca de la COVID-19. ${ }^{37}$ Así mismo, De Stefani et al. ${ }^{28}$ encontraron que el $70.2 \%$ de los encuestados 
tenía conocimiento acertado sobre los síntomas de COVID-19, pero aunque el 64.3\% declaró haber tenido entrenamiento en procedimientos de prevención de infecciones, el $48.7 \%$ refirió no tener conocimiento específico para prevenir el contagio de la COVID-19. Kamate SK et al. ${ }^{31}$ encontraron en un estudio multinacional que el $92.7 \%$ de los encuestados dijeron tener buen conocimiento del tema. Por el contrario, Singh Gambhir Ret al. ${ }^{29}$ encontraron notables deficiencias en el conocimiento de aspectos vitales de la COVID-19 y aunque más del $80 \%$ de los que respondieron la encuesta conocían del modo de transmisión y síntomas de la COVID-19, un tercio de los sujetos no conocían sobre el uso de EPP o sobre el tratamiento de la COVID-19.

Respecto al contagio de la COVID-19 entre los odontólogos, en el periodo de la encuesta (19 de junio al 24 de julio del 2020), 458 odontólogos refirieron haberse hecho la prueba de la COVID-19, de los cuales solo 33 odontólogos respondieron haber resultado positivos en la prueba, lo que corresponde al 0.6\% de la muestra total. Estos datos concuerdan con lo reportado por el INS para el 24 de julio en el país, donde se reportaban 34 odontólogos contagiados de COVID-19. ${ }^{16}$ También hay que resaltar que al 21 de septiembre del 2020 este reporte de contagios subió a 90 odontólogos con prueba de COVID-19 positiva en Colombia.

Otro hallazgo importante en nuestro estudio, es que de 5,370 odontólogos encuestados a 4,915 (91.53\%) no se les habían practicado pruebas de COVID-19, a pesar de que el 20\% refirió haber presentado algún síntoma de la COVID-19. Esto sugiere que a pesar de que los odontólogos son considerados de alto riesgo, las autoridades gubernamentales no los están teniendo en cuenta en los planes de testeo. Es importante que el gobierno no solo contemple planes restrictivos de trabajo para los odontólogos, sino que también cree planes adicionales como el testeo masivo - que ha demostrado ser una herramienta eficaz de prevención de contagio ${ }^{44}$ - entre la comunidad odontológica, apoyo de las ARL y a las EPS para prestar servicios de prevención efectivos y oportunos que den la seguridad a todo el sector incluyendo a los TSO y también a sus pacientes y familias.

Los odontólogos encuestados también refirieron que la infección de la COVID-19 es un riesgo para ellos (69.96\%), mayor que ir al supermercado (58.98\%). Sin embargo, el 64.32\% dijo tener confianza en que no se contagiará por COVID-19 durante la actividad clínica. Lo que sugiere que, aunque los odontólogos en Colombia están conscientes del riesgo de contagio del virus SARS-CoV-2, son optimistas en que no 
van a contagiarse en sus consultas. Estos resultados pueden deberse a características culturales o también a la confianza que genera la alta adherencia al seguimiento de protocolos de bioseguridad encontrada y al uso de EPP por los odontólogos en Colombia. Resultados similares en diferentes países han sido encontrados por otros autores en cuanto a percepción del riesgo de contagio de la COVID-19, durante las actividades clínicas en salud oral, como Putrino et al. ${ }^{30}$ en Italia donde $87.5 \%$ de los encuestados reportaron las actividades dentales como un riesgo de contagio, Duruk et al. ${ }^{37}$ encontró el $95 \%$ en Turquía y Ahmed MA et $a^{36}$ encontraron que el $90 \%$ de los encuestados creen que pueden contagiarse. De Stefani et al. ${ }^{28}$ concluyen que los odontólogos italianos consideran que la infección por el virus del SARS-CoV-2 es altamente peligrosa y no están confiados de ejercer sus actividades clínicas de forma segura.

Durante la pandemia de la COVID-19 el gobierno Colombiano ha creado una serie de beneficios para el personal de salud como médicos y enfermeras por considerarlos trabajadores esenciales. ${ }^{45}$ Sin embargo, no ha contemplado a los TSO dentro de estos planes, por lo que es muy importante que también la salud oral de los colombianos sea considerada como un servicio esencial y los TSO sean tenidos en cuenta en planes de protección laboral, beneficios y planes de préstamos especiales que permitan el resurgimiento económico de todo el sector odontológico del país. El Ministerio de Salud ha declarado que una vez se consiga la vacuna contra el COVID-19 la prioridad de aplicación la tendrán los adultos mayores de 70 años, las personas con comorbilidades (diabetes, hipertensión, obesidad, cáncer, entre otras), y los trabajadores de la salud. ${ }^{46}$ Los TSO esperamos que el gobierno nos esté contemplando dentro de estos trabajadores de salud que recibirán estas vacunas.

\section{CONCLUSIONES}

Un total de 5,370 odontólogos que trabajan en Colombia fueron escuchados sobre el impacto de la COVID-19 en su práctica clínica. El impacto encontrado fue alto en todos los aspectos estudiados, se observaron modificaciones en la práctica clínica, uso de EPP; desempeño laboral, planes futuros y percepción de riesgo de contagio. El uso reportado de EPP para la atención clínica y la adherencia a protocolos de bioseguridad de los odontólogos en Colombia es muy alto, llegando casi al 100\%. El contagio auto-reportado de COVID-19 entre los odontólogos fue bajo (0.6\%). 


\section{RECOMENDACIONES}

Se recomienda continuar investigando sobre el impacto de la pandemia en otros elementos de la vida personal, académica, profesional y laboral de los odontólogos, así como explorar la situación de salud bucal en diferentes grupos sociales en el marco de las medidas de aislamiento social. De igual forma, se requieren políticas y estrategias en el ámbito laboral que permitan por una parte mejorar la calidad de vida de los odontólogos, ya que dentro de los retos para la profesión está en seguir ofreciendo un servicio seguro, de calidad y rentable, ya que la pandemia ha originado una carta de costos adicionales reflejados en el aumento de los EPP, y los demás requisitos exigidos para brindar atención a los pacientes.

\section{AGRADECIMIENTOS}

Los autores agradecen a todas las Asociaciones y Agremiaciones que apoyaron la divulgación y respuesta de la encuesta: Federación Odontológica Colombiana (FOC), Asociación Colombiana de Facultades de Odontología (ACFO), Sociedad Colombiana de Ortodoncia (SCO), Asociación Colombiana de Odontología Estética y Digital (ACOED), Colegio Colombiano de Odontólogos (CCO), Asociación Colombiana de Endodoncia (ACE), Asociación Colombiana de Operatoria Dental Estética y Biomateriales (ACODEB), Academia Colombiana de Odontopediatría (ACOP), Asociación Colombiana de Radiología Oral y Maxilofacial (ACROM), Asociación de Odontólogos Javerianos (AOJ), Asociación de Odontólogos del Colegio Odontológico Colombiano (ASOCOC), Asociación de Exalumnos Fundación Universitaria San Martin (ASOEXFUSM), Asociación de técnicos y tecnólogos dentales de Colombia (ATTEDCO), Asociación de Odontólogos Neurofocales de Colombia (ODNF), Sociedad Colombiana de Ortopedia Maxilar (ACOM), Asociación Colombiana de Prostodoncia (ACP), Asociación Odontológica Colombiana de Implantes (SOCl), SOS Odontología, Asociación Colombiana de Cirugía Oral y Maxilofacial ( ACCOMF), Asociación Colombiana de Patología Oral (ACPO), Academia Colombiana de Oseointegración (ACO), Asociación Colombiana de Periodoncia y Oseointegración (ACPO) y a la Asociación de Ortodoncistas de la Universidad de Antioquia. Así mismo agradecen a todas las Facultades de odontología, sus decanos, directores de posgrado, profesores y colegas que motivaron la participación en la encuesta. Por último, 
un agradecimiento muy especial a cada uno de los odontólogos que dedicó su tiempo para responder la encuesta por su invaluable aporte al gremio Odontológico.

\section{CONFLICTO DE INTERESES}

Uno de los autores es el editor general de la Revista Facultad de Odontología Universidad de Antioquia. Se garantizó transparencia en el proceso editorial en tanto delegó la responsabilidad de la revisión editorial y por pares en los miembros del equipo editorial de la revista.

\section{AUTOR DE CORRESPONDIENCIA}

Andrés A. Agudelo Suárez

alonso.agudelo@udea.edu.co, oleduga@gmail.com

Facultad de Odontología, Universidad de Antioquia

Calle $70 \# 52-21$ CP 050010

Medellín, Colombia

\section{REFERENCES}

1. Zhu N, Zhang D, Wang W, Li X, Yang B, Song J et al. A novel coronavirus from patients with pneumonia in China, 2019. N Engl J Med. 2020; 382: 727-33. DOI: https://doi.org/10.1056/nejmoa2001017

2. Yu J, Zhang T, Zhao D, Haapasalo M, Shen Y. Characteristics of endodontic emergencies during coronavirus disease 2019 outbreak in Wuhan. J Endod. 2020; 46(6): 730-5. DOI: https://dx.doi.org/10.1016\%2Fj.joen.2020.04.001 
3. Qasim-Agha O, Berryman R. Acute splenic artery thrombosis and infarction associated with COVID-19 disease. Case Rep Crit Care. 2020; 2020: 8880143. DOI: https://doi.org/10.1155/2020/8880143

4. Sinadinos A, Shelswell J. Oral ulceration and blistering in patients with COVID-19. Evid Based Dent. 2020; 21(2): 49. DOI: https://dx.doi.org/10.1038\%2Fs41432-020-0100-z

5. Cascella M, Rajnik M, Cuomo A, Dulebohn SC, Di Napoli R. Features, evaluation, and treatment of Coronavirus (COVID-19). In: Treasure Island (FL); 2020.

6. Hu X, Liu S, Wang B, Xiong H, Wang P. Management practices of emergency departments in general hospitals based on blockage of chain of infection during a COVID-19 epidemic. Intern Emerg Med. 2020; 15(8): 1545-52. DOI: https://doi.org/10.1007/s11739-020-02499-6

7. Landi F, Marzetti E, Sanguinetti M, Ciciarello F, Tritto M, Benvenuto F et al. Should face masks be worn to contain the spread of COVID-19 in the postlockdown phase? Trans R Soc Trop Med Hyg. 2020. DOI: https://dx.doi.org/10.1093\%2Ftrstmh\%2Ftraa085

8. Vizcaychipi MP, Shovlin CL, McCarthy A, Howard A, Brown A, Hayes M et al. Development and implementation of a COVID-19 near real-time traffic light system in an acute hospital setting. Emerg Med J. 2020; 37(10): 630-36.

9. Weiss PG, Li S-TT. Leading change to address the needs and well-being of trainees during the COVID-19 pandemic. Acad Pediatr. 2020; 20: 735-41. DOI: https://dx.doi.org/10.1016\%2Fj.acap.2020.06.001

10. Jovanović A, Klimek P, Renn O, Schneider R, Øien K, Brown J et al. Assessing resilience of healthcare infrastructure exposed to COVID-19: emerging risks, resilience indicators, interdependencies and international standards. Environ Syst Decis. 2020: 1-35. DOI: https://dx.doi.org/10.1007\%2Fs10669-020-09779-8

11. Choi H, Cho W, Kim MH, Hur JY. Public health emergency and crisis management: case study of SARS-CoV-2 outbreak. Int J Environ Res Public Health. 2020; 17(11). DOI: https://www.mdpi.com/1660-4601/17/11/3984\# 
12. Villani FA, Aiuto R, Paglia L, Re D. Covid-19 and dentistry: prevention in dental practice, a literature review. Int J Environ Res Public Health. 2020; 17(12): 1-12. DOI: https://dx.doi.org/10.3390\%2Fijerph17124609

13. Tysiąc-Miśta M, Dziedzic A. The attitudes and professional approaches of dental practitioners during the COVID-19 outbreak in Poland: a cross-sectional survey. Int J Environ Res Public Health. 2020; 17(13): 4703. DOI: https://dx.doi.org/10.3390\%2Fijerph17134703

14. Ternera-Pulido JH. Los servicios odontológicos en la fase pandémica y post pandémica del Covid19.

15. Johns Hopkins University \& Medicine, Coronavirus Resource Center. COVID-19 Dashboard by the Center for Systems Science and Engineering (CSSE) at Johns Hopkins University (JHU) [Internet]. Maryland, USA; 2020.

16. Instituto Nacional de Salud. Noticias coronavirus-casos [Internet]. Bogotá: Ministerio de Salud; 2020.

17. Colombia. Presidencia. Decreto Número 457. Boletín Oficial, n51264, (22 de marzo de 2020).

18. Colombia. Ministerio de Salud. Lineamiento de bioseguridad para la prestación de servicios relacionados con la atención de la salud bucal durante el periodo de la pandemia por SARS-COV2 (COVID-19). Bigitá: MinSalud; 2020.

19. Colombia. Ministerio de Salud. Limpieza y desinfección en servicios de salud ante la introducción del nuevo coronavirus (SARS-CoV-2) a Colombia. Bogotá: MinSalud Bogotá; 2020.

20. Acosta-Ortiz S, Amaris-Jiménez E, Arias-Ruiz S, Betancur-Gracía CA, Benavides-Díaz N, BorreroCastro IL et al. Consenso técnico sobre las condiciones de bioseguridad frente al SARS-CoV-2 y la prevención de la enfermedad COVID-19 en el Sector Salud. Colombia: Colectivo Colombiano de Médicos Especialistas en Medicina Laboral y del Trabajo; 2020. 
21. Colombia. Ministerio de Salud. Orientaciones para la restauración de los servicios de salud en las fases de mitigación y control de la emergencia sanitaria por COVID-19 en Colombia. Bogotá: MinSalud; 2020.

22. de Graaff J, van Amerongen WE, Mulder GR. Hygiene in dental practice. Part II: measures to reduce the risk of contamination. ASDC J Dent Child. 1988; 55(1): 56-63.

23. Webber LM. Bloodborne viruses and occupational exposure in the dental setting. SADJ. 2000; 55(9): 494-6.

24. Araujo MWB, Andreana S. Risk and prevention of transmission of infectious diseases in dentistry. Quintessence Int. 2002; 33(5): 376-82.

25. Siles-Garcia AA, Alzamora-Cepeda AG, Atoche-Socola KJ, Peña-Soto C, Arriola-Guillén LE. Biosafety for dental patients during dentistry care after COVID-19: a review of the literature. Disaster Med Public Health Prep. 2020: 1-6. DOI: https://dx.doi.org/10.1017\%2Fdmp.2020.252

26. Cabrera-Tasayco FP, Rivera-Carhuavilca JM, Atoche-Socola KJ, Peña-Soto C, Arriola-guillén LE. Biosafety measures at the dental office after the appearance of COVID-19: a systematic review. 2020: 1-5. DOI: https://doi.org/10.1017/dmp.2020.269

27. Cagetti MG, Cairoli JL, Senna A, Campus G. COVID-19 outbreak in North Italy: an overview on dentistry: a questionnaire survey. Int J Environ Res Public Health. 2020; 17(11): 3835. DOI: https://dx.doi.org/10.3390\%2Fijerph17113835

28. De Stefani A, Bruno G, Mutinelli S, Gracco A. COVID-19 outbreak perception in Italian dentists. Int J Environ Res Public Health. 2020; 17(11): 3867. DOI: https://doi.org/10.3390/ijerph17113867

29. Singh-Gambhir R, Singh-Dhaliwal J, Aggarwal A, Anand S, Anand V, Kaur-Bhangu A. Covid-19: a survey on knowledge, awareness and hygiene practices among dental health professionals in an Indian scenario. Rocz Panstw Zakl Hig. 2020; 71(2): 223-9. DOI: https://doi.org/10.32394/rpzh.2020.0115 
30. Putrino A, Raso M, Magazzino C, Galluccio G. Coronavirus (COVID-19) in Italy: knowledge, management of patients and clinical experience of Italian dentists during the spread of contagion. BMC Oral Health. 2020; 20: 200.

31. Kamate SK, Sharma S, Thakar S, Srivastava D, Sengupta K, Hadi AJ et al. Assessing knowledge, attitudes and practices of dental practitioners regarding the covid-19 pandemic: a multinational study. Dent Med Probl. 2020; 57(1): 11-7. DOI: https://doi.org/10.17219/dmp/119743

32. Campus G, Diaz-Betancourt M, Cagetti MG, Carvalho JC, Carvalho TS, Cortés-Martiniorena JF et al. Study protocol for an online questionnaire survey on symptoms/signs, protective measures, level of awareness and perception regarding COVID-19 outbreak among dentists: a global survey. Int. J Environ Res Public Health. 2020; 17(15): 5598. DOI: https://dx.doi.org/10.3390\%2Fijerph17155598

33. Ternera-Pulido JH. Un complejo escenario laboral para la odontología en Colombia. Colombia: ECOE ediciones; 2016.

34. Berdejo-Casas JP, Ternera-Pulido JH. Estado de la afiliación a la seguridad social en salud de los odontólogos registrados ante la Secretaría Distrital de Salud entre 1960 y 2015, y estado de afiliación a la seguridad social en salud de los odontólogos habilitados como profesionales independientes; 2019.

35. Colombia. Ministerio de Salud. Estimaciones de profesionales de Odontología en Colombia por municipio 2019. Bogotá, MinSalud; 2020.

36. Ahmed MA, Jouhar R, Ahmed N, Adnan S, Aftab M, Zafar MS et al. Fear and practice modifications among dentists to combat novel coronavirus disease (COVID-19) outbreak. Int J Environ Res Public Health. 2020; 17(8): 2821. DOI: https://doi.org/10.3390/ijerph17082821

37. Duruk G, Gümüşboğa ZŞ, Çolak C. Investigation of Turkish dentists' clinical attitudes and behaviors towards the COVID-19 pandemic: a survey study. Braz Oral Res. 2020; 34: e054. DOI: https://doi.org/10.1590/1807-3107bor-2020.vol34.0054 
38. Empleo y desempleo [Internet]. Bogotá: DANE; [2020]. Available in https://www.dane.gov.co/index.php/estadisticas-por-tema/mercado-laboral/empleo-ydesempleo

39. FDI. Visión 2020 de la FDI. Suiza: World Dental Federation - FDI; 2020. Available in https://www.fdiworlddental.org/es/resources/brochures/vision-2020-de-la-fdi

40. Colectivas IY, La P, Para LDEB, et al. DURANTE EL PERIODO DE LA PANDEMIA POR SARS-COV-2 ( COVID-19 ) Ministerio de Salud y Protección Social Bogotá , mayo de 2020. Minist. salud y Prot. Soc. 2020;2:1-23.

41. Asociacion Colombiana de Facultades de Medicina (ASCOFAME). Protocolo para el retorno seguro a las actividades académico-administrativas en el campus universitario y en los sitios de práctica. Bogotá: ASCOFAME; 2020.

42. Colombia. Ministerio de Salud. Lineamientos, orientaciones y protocolos para enfrentar la COVID-19 en Colombia. Bogotá, MinSalud; 2020.

43. Cooperativa SCO life. Costos de tratamiento de ortodoncia 2020 [Interne]. Bogotá: Sco Life; 2020.

44. Gollier C, Gossner O. Group testing against Covid-19. Eur Netw Econ Fisc Policy Res. 2020; 3: 14.

45. Decreto Legislativo del 12 de abril del 2020. Ministerio de Salud y Protección Social, $n^{\circ} 538$.

46. Iván Duque anuncia quiénes recibirán primero la vacuna contra la covid-19. Revista Semana [Internet] 2020. Available in https://www.semana.com/nacion/articulo/quienes-recibiran-lavacuna-primero-en-colombia-segun-duque--vacuna-coronavirus/691158/ 